\title{
CRISPR-Cas9-Mediated Silencing of CD44 in Human Highly Metastatic Osteosarcoma Cells
}

\author{
Tang Liu ${ }^{a, b, c, d}$ Zuyun Yan ${ }^{a}$ Yong Liu ${ }^{d}$ Edwin Choy ${ }^{c}$ Francis J. Hornicek ${ }^{b}$ \\ Henry Mankinc Zhenfeng Duan ${ }^{b}$
}

\begin{abstract}
aDepartment of Orthopedics, the $2^{\text {nd }}$ Xiangya Hospital of Central South University, Changsha, China; bSarcoma Biology Laboratory, Department of Orthopaedic Surgery, David Geffen School of Medicine at University of Los Angeles, Los Angeles, CA, USA; 'Department of Orthopaedic surgery, Massachusetts General Hospital and Harvard Medical School, Boston, USA; 'State Key Laboratory of Powder Metallurgy, Central South University, China
\end{abstract}

\section{Key Words \\ Osteosarcoma $•$ CRISPR-Cas9 $•$ Meta-analysis $・$ CD44 $•$ Metastases $•$ Gene therapy}

\begin{abstract}
Background/Aims: Metastasis is the major cause of death in patients with osteosarcoma. There is an urgent need to identify molecular markers that promote metastasis. Cluster of differentiation 44 is a receptor for hyaluronic acid $(\mathrm{HA})$ and $\mathrm{HA}$-binding has been proven to participate in various biological tumor activities, including tumor progression and metastasis. Methods: We performed a meta-analysis to investigate the relationship between CD44 expression, survival, and metastasis in patients with osteosarcoma. We then utilized the CRISPR-Cas9 system to specifically silence CD44 in highly metastatic human osteosarcoma cells (MNNG/HOS and 143B) and further determined the functional effects of CD44 knockout in these cells. Results: The meta-analysis demonstrated that a high level of CD44 may predict poor survival and higher potential of metastasis in patients with osteosarcoma. The expression of CD44 in highly metastatic human osteosarcoma cell lines was efficiently blocked by CRISPR-Cas9. When CD44 was silenced, the proliferation and spheroid formation of these osteosarcoma cells was inhibited under 3-D culture conditions. Furthermore, the migratory and invasive functions were also impaired in these highly metastatic osteosarcoma cells. Conclusion: These results suggest that developing new strategies to target CD44 in osteosarcoma may prevent metastasis and improve the clinical outcome of osteosarcoma patients.

(C) 2018 The Author(s)

Published by S. Karger AG, Basel
\end{abstract}

\section{Introduction}

Osteosarcoma is a common malignant primary tumor of bone, which mainly affects children and adolescents [1,2]. Current treatment for osteosarcoma involves surgical resection and multi-agent neoadjuvant and adjuvant chemotherapy [1-3]. At the time of

Department of Orthopedics, the 2nd Xiangya Hospital of Central South University, 139 Renmin Road, Changsha, Hunan (China)

E-Mail liutang1204@csu.edu.cn, zduan@mednet.ucla.edu

\section{KARGER}


diagnosis, up to $15-20 \%$ of patients may have detectable metastatic disease [4]. Metastatic osteosarcoma usually has unsatisfactory rates of response to the current standard chemotherapy, resulting in poor prognosis [4]. The 5-year survival rate for patients with aggressive metastases is only about 10-30\% [5]. The cellular and molecular mechanisms underlying metastasis formation in osteosarcoma remain unclear.

Cluster of differentiation 44 (CD44) is a receptor for hyaluronic acid (HA), and CD44 bound to HA has been proven to participate in various biological tumor activities, including tumor progression, metastasis, and chemoresistance [6-8]. Though the biological relevance of CD44 expression in osteosarcoma progression and metastasis has been reported, the results are controversial. A previous study has noted that CD44 expression could not predict the prognosis for patients with osteosarcoma [9]. Another study has also suggested that CD44 expression is not associated with the overall survival rate or metastasis in osteosarcoma [10]. However, in a study by Kim et al., the metastatic and prognostic value of CD44 for patients with osteosarcoma has been reported [11]. In our previous studies, we have confirmed that CD44 is overexpressed in metastatic and recurrent osteosarcoma tissues, and that upregulation of CD44 is correlated with poor chemotherapy response and unfavorable clinical outcome [6]. These data suggest that further study is required to clarify the prognostic value of CD44 in osteosarcoma.

Meta-analysis uses a statistical approach that systematically combines the results from previous multiple research studies to derive conclusions about a certain body of research [12]. Results from a meta-analysis may include a more precise estimate of risk factors (such as the expression of CD44), the effect of the treatment, or other outcomes, providing more valuable data than an individual study contributing to the pooled analysis.

In addition, the functional role of CD44 expression in cell migration and invasion of osteosarcoma is also still largely unknown. Recently, the clustered regularly interspaced short palindromic repeats (CRISPR)-associated Cas9 (CRISPR-Cas9) system has been implemented in a multitude of model organisms and cell types [13-15]. The CRISPR-Cas9 system uses Cas9, which complexes with single guided RNA (sgRNA), to cleave DNA 3-4 base pairs upstream of a protospacer-adjacent motif (PAM) and generate double-strand breaks (DSBs) in a sequence-specific manner $[14,15]$. It is evident that the CRISPR-Cas9 genome editing technology has revolutionized the field of genetic engineering and holds the potential to overcome many of the limitations of earlier techniques to carry out deletions, insertions, translocations, and inversions at specific sites in the DNA of cells [13-15].

Due to the controversial discussions on the relevance of CD44 expression in osteosarcoma biology and the limited knowledge on CD44 biological functions in osteosarcoma and metastasis, in this study, we further explore the correlation between CD44 expression and osteosarcoma prognosis through a meta-analysis of published case-control studies. We then adopted the CRISPR-Cas9 system to specifically silence CD44 at the DNA level in human highly metastatic osteosarcoma cells (MNNG/HOS and 143B), and further determined the effects of $C D 44$ knockout on function in human highly metastatic osteosarcoma cells.

\section{Materials and Methods}

\section{Meta-analysis}

The meta-analysis was conducted in accordance with the standard guidelines of the Preferred Reporting Items for Systematic Reviews and Meta-Analyses (PRISMA) 2009 Checklist (http://www.prismastatement. org/statement.htm) and Meta-analysis of Observational Studies in Epidemiology group (MOOSE) [16].

Identification of relevant studies. Electronic searches on PubMed, Embase, and Web of Science databases were conducted to collect relevant literatures for this meta-analysis. The last search update was performed on October 30, 2017, without any language restrictions. The search strategy included the following sets of key words and their combination search terms: "CD44", "osteosarcoma OR malignant bone tumor OR malignant bone cancer", and "survival OR prognosis OR metastasis OR outcome". We also performed a manual search in order to identify other potentially eligible studies. 
The most complete study was included in our analysis when there was more than one study containing overlapping data from the same authors. Requests were emailed to the authors when supplementary information and essential data were needed.

Two independent reviewers first searched potentially relevant studies by reading the titles and abstracts, and then explored further by reading the full texts and assessing for inclusion. Another two senior reviewers read through the extracted articles a second time for confirmation. Disagreements were resolved by discussion among these reviewers and consultation with a third senior reviewer.

Eligibility criteria. Studies were considered eligible according to following criteria: (i) patients with osteosarcoma was studied; (ii) the expression of CD44 and variant isoforms of CD44 (CD44V) in tumor tissue or blood sample were measured; (iii) the associations between CD44 expression and prognostic outcome (metastasis or death) of patients were investigated; and (iv) sufficient data was provided to estimate risk ratios (RR) and its 95\% confidence intervals (95\% CI) for survival rates.

Articles were excluded if they met the following criteria: (i) reviews, case reports, comments, economic analyses, conference abstracts, animal studies, and laboratory studies; (ii) studies of non-dichotomous CD44 expression levels; or (iii) lack of crucial information about survival outcome or could not estimate RR and $95 \%$ CI using the available data.

Quality assessment. Two investigators critically assessed the quality of all the included studies based on the critical guidelines of the Dutch Cochrane Centre proposed by MOOSE for prognostic meta-analysis. The key points of the review checklist included the following: (i) clear description of study population and origin of country; (ii) clear definition of diagnosis of osteosarcoma; (iii) clear explanation of study design; (iv) clear description of outcome assessment; (v) clear report of CD44 measure method; (vi) clear definition of cut-off of CD44; and (vii) sufficient follow-up period. We excluded studies that did not specify any one of the aspects above so as not to compromise the quality of the meta-analysis.

Data extraction, conversion and analysis. The two investigators independently extracted relevant information in standardized data collection forms to rule out any discrepancy. Disagreements were resolved by discussion in a meeting that included experts from the department. The following characteristics of the individual eligible research articles were collected: name of the first author, year of publication, origin of population, case number, sample source, detection methods, cut-off values, and prognostic outcome.

RRs with their 95\% CIs were extracted according to the following methods [17]. Reported analysis results for survival and metastasis in the included studies were considered eligible for the meta-analysis. In most instances, the total number of observed deaths or metastatic cases and the number of samples in each group or other valuable data provided by the authors were extracted to calculate RRs. In the absence of RRs and 95\% CIs, if only Kaplan-Meier curves were available, data were extracted from the graphical survival plots to estimate the RRs following the previously described method $[17,18]$. If needed, we sought original data directly from the authors of the relevant studies. All the results extracted according to the above methods were compared, and disagreements were discussed until a consensus was reached among all the authors. The pooled RRs with their 95\% CIs and $P$ values were reported as the results, with a RR $>1$ being associated with elevated risk of mortality or metastasis.

The prognostic outcome of osteosarcoma associated with CD44 expression was estimated by using the RR and their associated 95\% CI for each study. RRs with 95\% CIs were used to combine the pooled data. Heterogeneity of combined RRs was assessed by Cochran's Q test and Higgin's I² statistic [19, 20]. Heterogeneity was considered statistically significant at $P<0.05$ or $\mathrm{I}^{2}>50 \%$. Pooled RR was calculated using a fixed effect model or random effect model to evaluate the relationship between CD44 expression and survival rate. A fixed effect model (Mantel-Haenszel test) was applied in the absence of between-study heterogeneity $\left(P \geq 0.05\right.$ or $\left.\mathrm{I}^{2} \leq 50 \%\right)$ [21], while the random effect model (Der Simonian and Laird method) was applied if significant heterogeneity was observed $\left(P<0.05\right.$ or $\left.\mathrm{I}^{2}>50 \%\right)$ [22].

The Begg's funnel plot and Egger's bias indicator test were used to evaluate the potential publication bias among the included studies [23, 24]. $P<0.05$ in all the two-sided statistical tests was regarded as significant. No corrections were made for multiple comparisons. All analyses were conducted using the Stata package version 12.0 (Stata Corporation, College Station, Texas, USA).

Related basic research

Human osteosarcoma cell lines. The osteosarcoma cell lines MNNG/HOS and 143B used in this study were purchased from ATCC (Manassas, VA, USA). The two cell lines are derivatives from the same osteosarcoma 
patient [25]. The MNNG/HOS cell line was transformed with N-methyl-N'-nitro-N-nitrosoguanidine (MNNG) [26], while the 143B cell line was transformed with Ki-ras [27]. When the two cell lines were injected into the proximal tibia of athymic nude mice, both MNNG/HOS and 143B cells were able to form tumors with high efficiency, and the injected mice could develop pulmonary metastases [28]. The two cell lines were cultured in RPMI 1640 (Life Technologies, Grand Island, NY, USA) supplemented with 10\% fetal bovine serum (FBS), 100 units $/ \mathrm{mL}$ penicillin, and $100 \mu \mathrm{g} / \mathrm{mL}$ streptomycin (Life Technologies). Cells were incubated at $37^{\circ} \mathrm{C}$ in $5 \% \mathrm{CO}_{2}-95 \%$ air atmosphere and passaged when near-confluent monolayers were achieved using trypsinEDTA solution.

CRISPR-Cas9 plasmid design and purification. The CRISPR-Cas9 and green fluorescent protein (GFP) fusion protein expression vector U6gRNA-Cas9 $\pm 2 \mathrm{~A}$-GFP guide by CD44 sgRNA (abbreviated as CD44Cas9-GFP) was purchased from Horizon Discovery (DNA 2.0 Inc., CA, USA). GFP is co-expressed from the same mRNA as the Cas9 protein via a $2 \mathrm{~A}$ peptide linkage, which enable tracking of transfection efficiency. The exon of $C D 44$ selected for sgRNA design is located on the first coding exon (Fig. 1). The CD44 sgRNA sequence is as follows: 5'-CCGATCTGCGCCAGGCTCAG-3' (Fig. 1). The pEGFP-N3 plasmid was purchased from Clontech Laboratories, Inc. (Mountain View, CA, USA). Plasmid was purified using QIAGEN Plasmid Mega Kits (Hilden, Germany) according to the Plasmid Purification Handbook. To determine the yield of plasmid, DNA concentrations were determined by both UV spectrophotometry at $260 \mathrm{~nm}$ and quantitative analysis on an agarose gel.

Work flow of Lipofectamine-mediated transfection of CD44 sgRNA-Cas9-GFP. Transfection of CD44 sgRNA-Cas9-GFP into MNNG/HOS and 143B cells were performed with Lipofectamine ${ }^{\circledR} 3000$ Reagent (Life Technologies) according to the manufacturer's instructions. Briefly, MNNG/HOS and 143B cells were seeded in 12 -well plates at a density of $1.0 \times 10^{5}$ cells $/ \mathrm{mL}, 1 \mathrm{~mL}$ of cells per well. After $24 \mathrm{~h}$, we used Opti-MEM ${ }^{\circledR}$ Medium to rinse the cells three times, and then added $1 \mathrm{~mL}$ serum-free medium for cell culturing. Then, $1.5 \mu \mathrm{l}$ Lipofectamine ${ }^{\circledR} 3000$ Reagent was diluted in $50 \mu \mathrm{l}$ Opti-MEM ${ }^{\circledR}$ Medium, a master mix of DNA was prepared by diluting $1 \mu \mathrm{g}$ DNA in $50 \mu \mathrm{l}$ Opti-MEM ${ }^{\circledR}$ Medium and adding $2.0 \mu \mathrm{l} \mathrm{P3000}{ }^{\mathrm{TM}}$ Reagent. Following, diluted DNA was added to the tube of diluted Lipofectamine ${ }^{\circledR} 3000$ Reagent (1:1 ratio). After incubation for $5 \mathrm{~min}$ at room temperature, the DNA-lipid complex was added to the cells. After incubation for $48 \mathrm{~h}$, the positive cells successfully transfected with CD44 sgRNA-Cas9-GFP plasmid were sorted with flow cytometry and were cultured and expanded for further study. Untransfected cells were used as control.

Fluorescencemicroscopeobservation. To observe the transfection efficacy of CD44-Cas9-GFP or pEGFP-N3 plasmid expression into MNNG/HOS and 143B, fluorescence analysis was performed by investigating the GFP expression levels in the transfected cells. Briefly, MNNG/HOS and 143B cells were seeded in 12well plates at a density of $1.0 \times 10^{5}$ cells $/ \mathrm{ml}, 1 \mathrm{~mL}$ of cells per well. Then, the cells were transfected with CD44-Cas9-GFP or pEGFP-N3 plasmid. After incubation for $24 \mathrm{~h}$ and $48 \mathrm{~h}$, detection was performed under fluorescence. Osteosarcoma cells were then visualized on a Nikon Eclipse Ti-U fluorescence microscope (Nikon Instruments, Inc., NY) equipped with a SPOT RT digital camera from Diagnostic Instruments, Inc. (Sterling Heights, MI).

Western blotting. After transfection of CD44-Cas9-GFP plasmid, the expression of CD44 protein in MNNG/HOS and 143B cells was evaluated by Western blotting. Protein lysates from osteosarcoma cells were extracted using 1× RIPA Lysis Buffer (Upstate Biotechnology, Charlottesville, VA, USA). Protein concentrations were determined by Protein Assay Reagents (Bio-Rad, Hercules, CA, USA) and a SPECTRAmax Microplate Spectrophotometer from Molecular Devices (Sunnyvale, CA, USA). The primary antibodies for CD44 (1:1000 dilution), and Cas9 (1:1000 dilution) were purchased from Cell Signaling Technology (Danvers, MA, USA). Secondary antibodies IRDye ${ }^{\mathrm{R}} 800 \mathrm{CW}$ or IRDye ${ }^{\mathrm{R}} 680 \mathrm{LT}$ were purchased from LI-COR Biosciences (Lincoln, NE, USA). Western blotting analyses were carried out as previously described [6, 29]. Membrane signals were scanned using the Odyssey Infrared Imaging System and analyzed using Odyssey 3.0 software (LI-COR Biosciences, NE, USA). Relative expression values were normalized assigning the value of the cells in control groups to 1.0 .

Wound healing assay. The effect of CD44 knockout in osteosarcoma cells on migration was evaluated by wound healing assay. Briefly, MNNG/HOS cells with or without transfection of pEGFP-N3, MNNG/HOSCD44-Cas9-GFP cells, 143B cells with or without transfection of pEGFP-N3, and 143B-CD44-Cas9-GFP cells were seeded into 12 -well culture plates at a density of $1.0 \times 10^{5}$ cells per well. When the cells reached 80 $100 \%$ confluence, three parallel lines with similar width were then created in each well using sterile $200 \mu \mathrm{l}$ pipette tips and rinsed to clear cell debris and suspension cells. Fresh regular RPMI 1640 medium was 


\section{Cellular Physiology Cell Physiol Biochem 2018;46:1218-1230

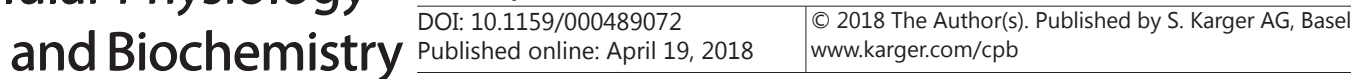

Liu et al.: Silence CD44 in Osteosarcoma Cells

Fig. 1. (A) Schematic of U6 CD44 sgRNA-CMV Cas9GFP expression cassette in the single plasmid system. GFP is co-expressed from the same mRNA as the Cas 9 protein via a 2A peptide linkage, which enables tracking of transfection efficiency. The exon of CD44 selected for guide RNA design is located on the first coding exon. The human U6 promoter is used to drive sgRNA expression, while the CMV promoter drives expression of Cas9 and GFP proteins. The position of the frameshift that CRISPR-Cas9 knocks out is located at the first exon within the CD44 gene (NM 000610.3_CD44). (B) Schematic structure of CRISPRCas9 system functions on targeting the CD44 gene. The core components of the CRISPR-Cas9 system are a nuclease Cas 9 and a single guided RNA (sgRNA). The CRISPR-Cas9 system uses Cas9, which complexes with sgRNA to cleave target DNA and generate dou-

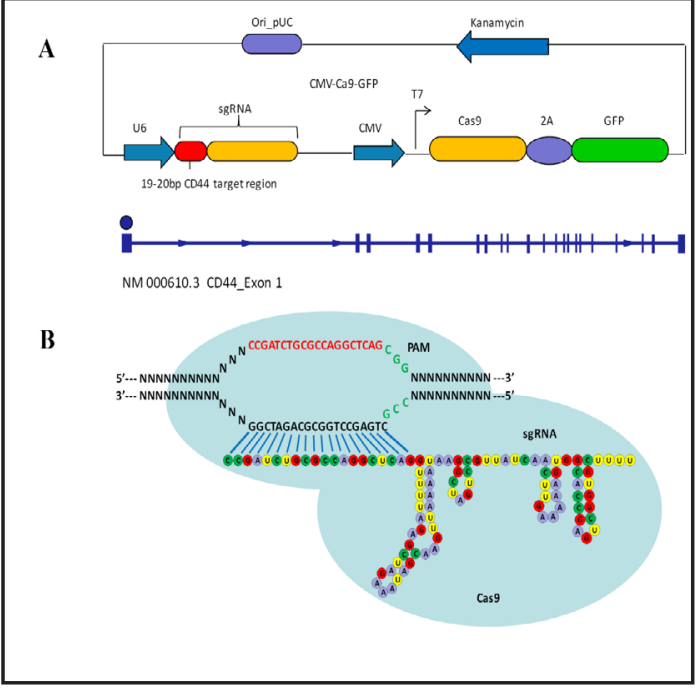
ble-strand breaks (DSBs) in a sequence-specific man-

ner about 3-4 base pairs upstream of a protospacer adjacent motif (PAM). The red font is the 20 bp guide sequence for sgRNA, and AGG (green font) is the sequence of PAM. The black font in sgRNA sequence is the commonly used sequence for tracrRNA.

added and the cells were allowed to close the wound for 24 hours. Three images were captured per well at different time points after wounding $(0,12$, and 24 hours $)$ to monitor the repair process by a microscope (Nikon) at $100 \times$ magnification. The width of the wound was measured at 10 sites in each image. The cell migration distance was calculated by subtracting the wound width at each time point from the wound width at the 0 -hour time point.

Matrigel invasion assay. Matrigel invasion assay examined the alteration of cell invasion activity using a BD BioCoat ${ }^{\mathrm{TM}}$ Matrigel $^{\mathrm{TM}}$ Invasion Chamber (Becton-Dickinson, MA, USA) following the manufacturer's recommendations. Specifically, $5 \times 10^{4}$ cells were added into the upper chamber of each well in medium without FBS or antibiotics, while the bottom chambers were filled with $500 \mu \mathrm{l}$ medium with $10 \%$ FBS without antibiotics. After a 24-hour incubation period, the non-invading cells were carefully scrubbed from the upper surface of the membrane with a cotton swab. Following the processes of fixation in $100 \%$ methanol and staining in hematoxylin and eosin, the invading cells were counted in three images of each membrane under a microscope using a $10 \times$ objective.

Determination of the CD44 knockout effect on spheroid formation in 3-Dimensional (3-D) culture of osteosarcoma cells. Cell spheroid formation was performed following HDP 1096 Perfecta3D $^{\mathrm{R}}$ 96-Well Hanging Drop Plates Protocol (3D Biomatrix). Initially, hanging drops were formed by pipetting $40 \mu \mathrm{l}$ of cell suspension $\left(2.5 \times 10^{5} / \mathrm{ml}\right)$ into each well containing MNNG/HOS, MNNG/HOS transfected with p-EGFP-N3, MNNG/HOS-CD44-Cas9-GFP, 143B, 143B transfected with p-EGFP-N3, and 143B-CD44-Cas9-GFP, respectively. Five days later, the spheroids were harvested for further study from the bottom side of the plate by pipetting $100 \mu$ l Phosphate Buffer Solution (PBS) into each well gently without any destruction of the morphology of the spheroids. Finally, after incubating with $1 \mu$ M Calcein AM (Life Technologies, Grand Island, NY, USA) for $15 \mathrm{~min}$, the spheroids were photographed on a Nikon Eclipse Ti-U fluorescence microscope (Nikon Instruments, Inc., NY) equipped with a SPOT RT digital camera from Diagnostic Instruments, Inc. (Sterling Heights, MI).

\section{Statistical analysis}

GraphPad PRISM 5 software (GraphPad Software, La Jolla, CA) was used to statistically analyze the data. The differences between groups were also evaluated using the two-sided Student's $t$-test. Errors were SD of averaged results and $p$ values $<0.05$ were considered statistically significant between means, $p$ values $<0.01$ were accepted as a significant difference between means. 


\section{Results}

\section{Meta-analysis results}

Eligible studies. A total of 237 studies were identified after searching in PubMed (79 papers), Embase (85 papers), and Web of Science (73 papers) for publications on CD44 expression associated with prognosis of osteosarcoma. The titles, publication types, and abstracts were initially evaluated and the full texts were further reviewed. Finally, nine studies that met the inclusion criteria were considered to qualify for the present metaanalysis. Fig. 2 shows the flow diagram of candidate study selection in our study.

Characteristics of included studies. We collected the essential data from the enrolled nine studies, which were conducted between 1999 and 2017 [6, 9, 11, 30-35]. A total of 548 participants were analyzed. The sample size of the included studies ranged from 34 to 90 patients. The expression of CD44 was measured in collected cancer tissues in all the eligible studies. Immunohistochemistry (IHC) was widely applied to detect the expression level of CD44. Studies in this meta-analysis referred to the evaluation of CD44 expression for prognostic outcome in osteosarcoma, including seven studies for survival outcome and eight studies for metastasis outcome. The main features of these nine studies are summarized in Table 1.

Survival associated with CD44 expression in osteosarcoma. For studies evaluating survival outcome, a fixed effect model was applied to calculate the pooled RR and its $95 \%$ CI because non-significant heterogeneity had been found in the seven cohorts $\left(\mathrm{I}^{2}=39.8 \%, P=0.126\right)$. The result showed that high levels of CD44 may predict poor survival, with the pooled RR being 1.42 (95\% CI: 1.20-1.67, $P=0.000$ ) (Fig. 3A).

Metastasis associated with CD44 expression in osteosarcoma. The studies evaluating metastasis outcome, a fixed effect model was used to calculate the pooled RR with 95\% CI due to the non-significant heterogeneity $\left(\mathrm{I}^{2}=0.4 \%, P=0.426\right)$. The result showed that high levels of CD44 may predict higher potential of metastasis, with the pooled RR being 1.58 (95\% CI: 1.29-1.94, $P=0.000$ ) (Fig. 3B).

Publication bias and sensitivity analysis. Potential publication bias was assessed by Begg's funnel plot and Egger's test. Among the seven cohorts evaluating survival and the eight cohorts evaluating metastasis, there was no obvious asymmetry in Begg's funnel plots, and the Begg's test results also showed no potential publication bias (Fig.3C \& Fig. 3D, survival: $P=0.548>0.05$; metastasis: $P=1.000>0.05)$. The Egger's test results also showed no potential publication bias (survival: $\mathrm{t}=0.76, P=0.484>0.05$; metastasis: $\mathrm{t}=0.89, P=0.409>0.05$ ).

\section{Basic research results}

Transfection ofCD44 sgRNA-Cas9-GFP significantly inhibits CD44 expression. To determine the transfection efficiency of CD44-Cas9-GFP or pEGFP-N3 plasmid into MNNG/HOS and 143B cells, fluorescence expression was examined by a fluorescence microscope. As seen in Fig. 4A, MNNG/HOS and 143B cells were successfully transfected with CD44-Cas9-GFP or pEGFP-N3.

CRISPR-Cas9 precisely enables specific genomic locus manipulating by providing sgRNA. To evaluate whether CRISPR-Cas9 complexed with CD44 sgRNA could knock out CD44, Western blot was performed. The assay demonstrated that $C D 44$ protein expression was significantly inhibited in MNNG/HOS cells transfected with CD44-Cas9-GFP. CD44 expression of MNNG/HOS was repressed $10.13 \pm 2.46$

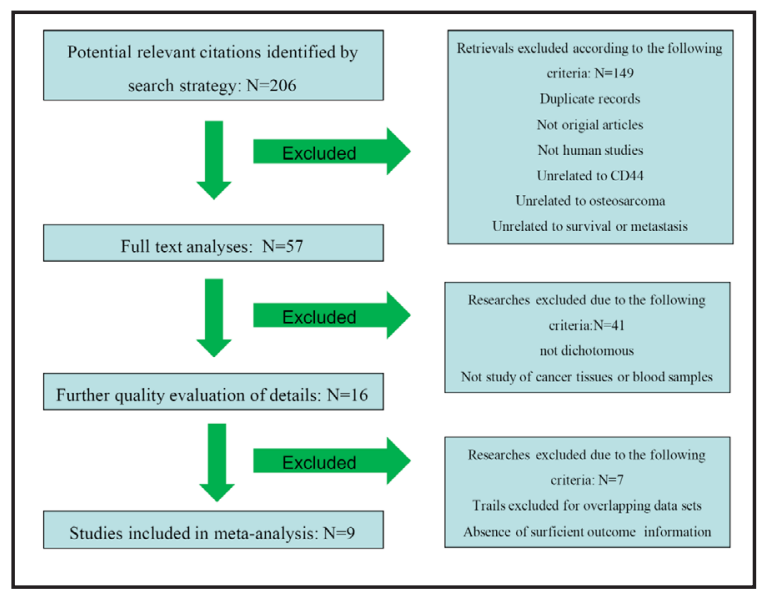

Fig. 2. Flow diagram of the study selection process. 
Table 1. Characteristics of the studies on CD44 included in this meta-analysis. IHC: Immunohistochemistry

\begin{tabular}{|c|c|c|c|c|c|c|c|c|}
\hline Author & Publication year & Cases & CD44 type & Sample & Test method & Cut-off & Survival Outcome & Metastasis Outcome \\
\hline Gao Yan 6 & 2015 & 70 & CD44 & tissue & IHC & Grade $\geq 2$ & YES & YES \\
\hline Gvozdenovic $\mathrm{A}^{30}$ & 2013 & 53 & CD44 & tissue & IHC & Grade $\geq 2$ & YES & YES \\
\hline Deng ZM ${ }^{31}$ & 2013 & 90 & CD44V6 & tissue & IHC & $\geq 25 \%$ & YES & YES \\
\hline Ma Qing32 & 2011 & 63 & CD44 & tissue & IHC & Scored(NA) & YES & YES \\
\hline Boldrini ${ }^{9}$ & 2010 & 34 & CD44 & tissue & IHC & $\geq 10 \%$ & YES & NA \\
\hline $\mathrm{Hu} \mathrm{WH}^{35}$ & 2009 & 87 & CD44V6 & tissue & $\mathrm{IHC}$ & Grade $\geq 3$ & NA & YES \\
\hline Peng TS 33 & 2002 & 62 & $\mathrm{CD} 44 \mathrm{~s}$ & tissue & IHC & $\geq 20 \%$ & NA & YES \\
\hline $\mathrm{Kim}^{11}$ & 2002 & 50 & CD44V5 & tissue & IHC & $\geq 50 \%$ & YES & YES \\
\hline Kuryu 34 & 1999 & 39 & CD44V6 & tissue & $\mathrm{IHC}$ & $\geq 10 \%$ & YES & YES \\
\hline
\end{tabular}

A

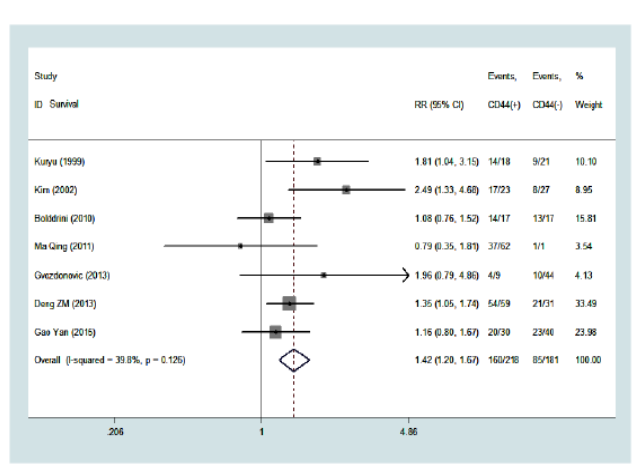

$\mathbf{B}$

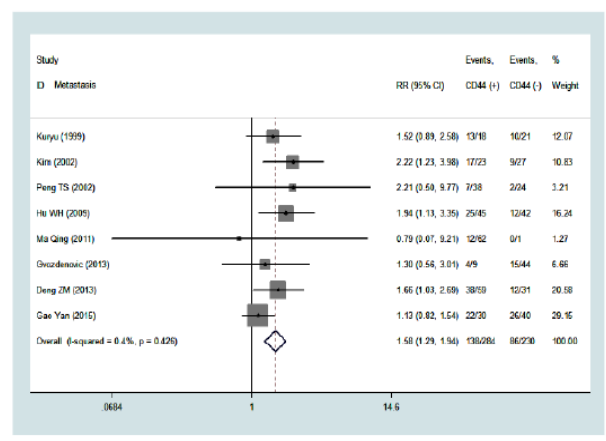

C

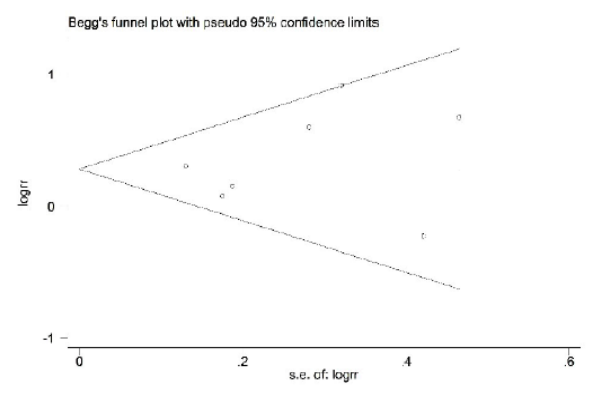

$\mathbf{D}$

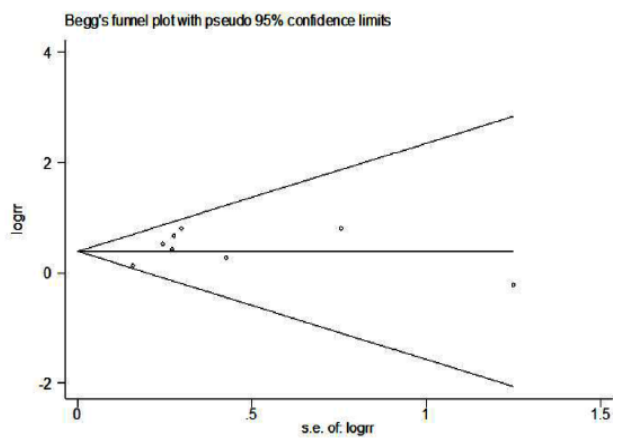

Fig. 3. (A) CD44 expression and overall survival rate of osteosarcoma patients. (B) CD44 expression and metastasis of osteosarcoma patients. (C) Among 7 cohorts evaluating survival, no obvious asymmetry was observed in Begg's funnel plots. (D) Among 8 cohorts evaluating metastasis, no obvious asymmetry was observed in Begg's funnel plots.

fold ( $\mathrm{p}<0.01$ ). In the 143B cells transfected with CD44-Cas9-GFP, CD44 expression was also significantly inhibited. CD44 expression of $143 \mathrm{~B}$ was repressed $9.56 \pm 1.79$ fold $(\mathrm{p}<0.01)$. Furthermore, as expected, Cas9 protein was expressed in MNNG/HOS and 143B cells transfected with CD44-Cas9-GFP (Fig. 4B). These data revealed that CD44 expression was efficiently repressed in osteosarcoma cell lines transfected with CD44-Cas9-GFP. 
Fig. 4. (A) Fluorescence analysis showed that GFP was detected in MNNG/HOS-pEGFP-N3, MNNG/ HOS-CD44-Cas9-GFP, 143-B-CD44Cas9-GFP, and 143-B-pEGFP-N3 cells, which suggests that MNNG/ HOS and 143-B cells were successfully transfected with CD44-Cas9GFP or pEGFP-N3. (B) Western blotting analysis confirmed that CD44 protein expression was significantly inhibited in MMNG/HOS and 143B cells transfected with C-

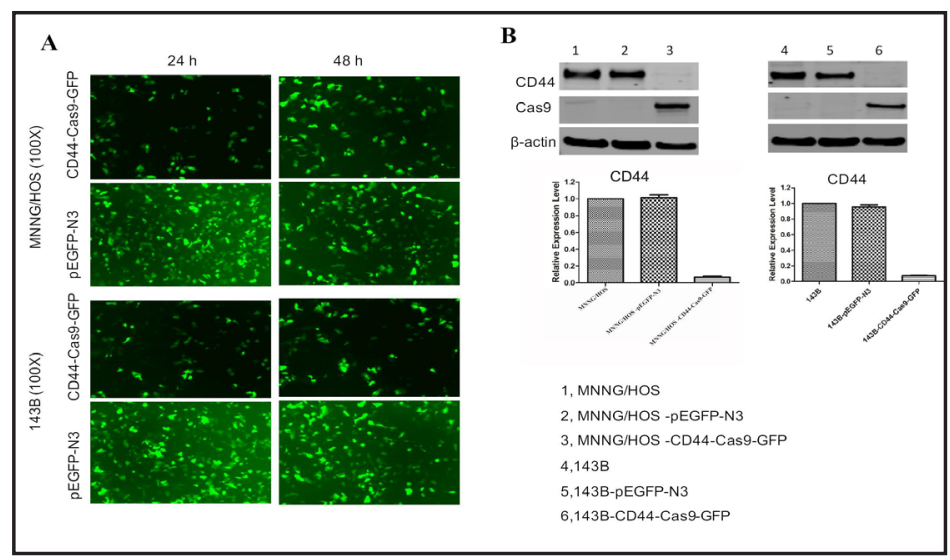
Cas9-GFP. Furthermore, Cas9 pro-

tein was expressed in MNNG/HOS and 143B cells transfected with CD44-Cas9-GFP. CD44 expression was repressed $10.13 \pm 2.46$ fold $\left({ }^{*} \mathrm{p}<0.01\right)$ and $9.56 \pm 1.79$ fold $\left({ }^{* *} \mathrm{p}<0.01\right)$ in MNNG/HOS and $143 \mathrm{~B}$ cells transfected with CD44-Cas9-GFP, respectively.

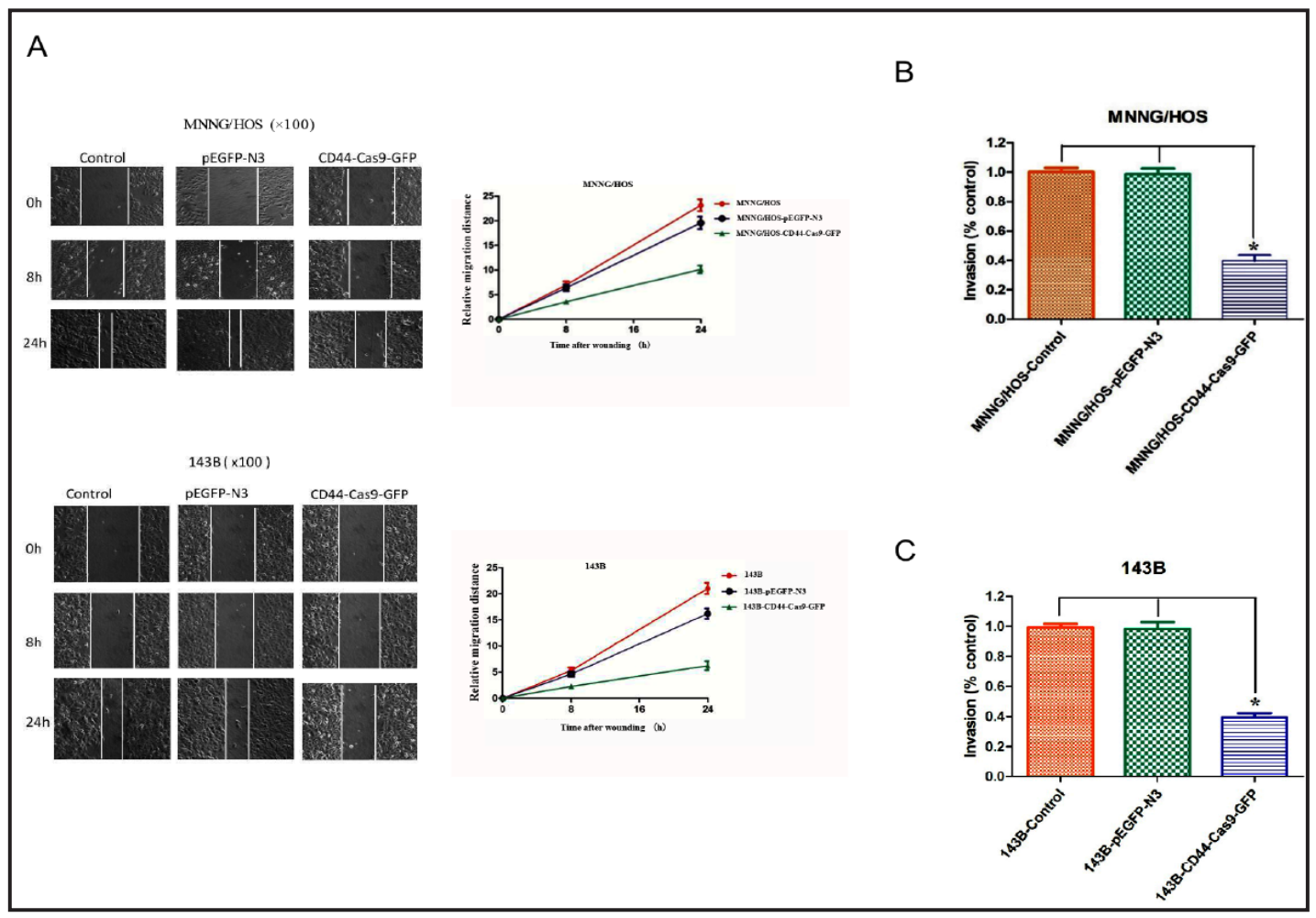

Fig. 5. In the wound healing assay (A), wounds were almost recovered after 24-hour migration in cells with or without transfection with pEGFP-N3. However, wound healing showed a substantial inhibition when the cells were transfected with CD44-Cas9-GFP. Transwell invasion assay (B, C) showed the average numbers of cells transfected with CD44-Cas9-GFP invading through the matrigel were significantly lower than that of the cells with or without transfection with pEGFP-N3. These assay was conducted in triplicate.

Knockout of CD44 by CRISPR-Cas9 suppressed the migration and invasion activity of osteosarcoma cells. Migration and invasion are crucial prerequisites for osteosarcoma metastasis. The wound healing showed a substantial inhibition when the cells were transfected with CD44-Cas9-GFP (Fig. 5). In transwell invasion assays, the average numbers 


\section{Cellular Physiology

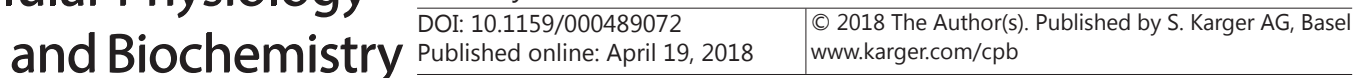

Fig. 6. Knockout of CD44 suppressed sphere formation of osteosarcoma cells in three-dimensional culture. Spheroid formation of different cells after 5-day culture and the relative diameters compared with untreated cells. The assay was conducted in triplicate.

of cells transfected with CD44-Cas9GFP invading through the Matrigel was significantly lower than that of the cells with or without transfection with pEGFP-N3 (Fig. 5). Taken together, the

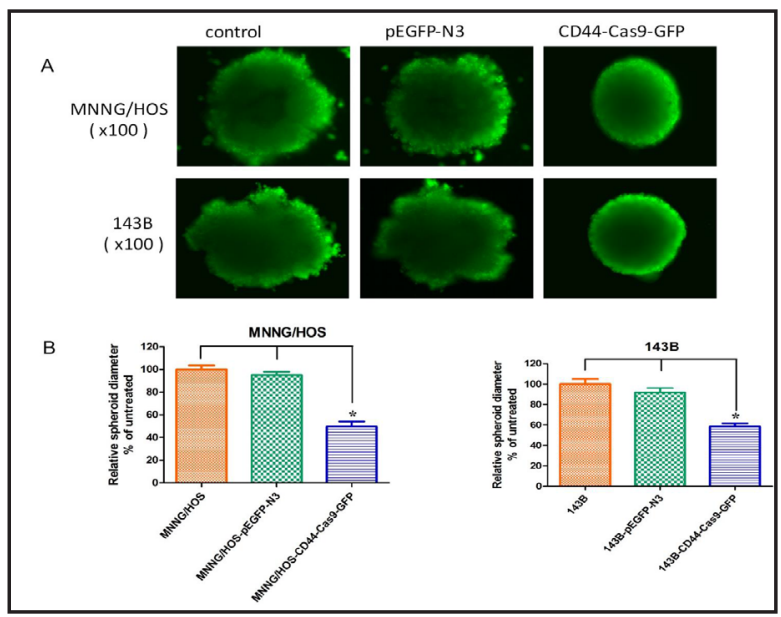
wound healing and the transwell invasion assay both demonstrated that knockout of $C D 44$ inhibited the migration and invasion capabilities of MNNG/HOS and 143B cells.

Knockout of CD44 by CRISPR-Cas9 inhibited the spheroid formation in 3-D culture of osteosarcoma cells. After a 5-day culture in the 3-D environment, which mimics the in vivo setting, the diameter of spheroids formed by cells transfected with CD44-Cas9-GFP was relatively smaller than other cell spheroids (Fig. 6). At the same time, the border of spheroids formed by cells transfected with CD44-Cas9-GFP was relatively smoother than other cell spheroids (Fig. 6). These results suggest that $C D 44$ enhances the invasion and migration of MNNG/HOS and 143B cells.

\section{Discussion}

In the present meta-analysis, we show that high expression of CD44 could predict poor survival and metastasis in patients with osteosarcoma. Interestingly, a meta-analysis conducted by Liu et al. reaches the opposite conclusion of that of our current study [10]. In their analysis, only six studies with 329 osteosarcoma patients were involved [10]. A metaanalysis on this subject was also performed on the CD44 splice variant CD44V6 by Zhang et al. and it was found that CD44V6 over-expression was associated with overall survival and metastasis [36]. In our meta-analysis, a total of nine studies with 548 participants were analyzed. The result of current meta-analysis is consistent with our previous study [6].

Admittedly, some limitations existed in this meta-analysis, although statistical results suggest that there is no publication bias. Osteosarcoma is rare bone tumor, the number of included studies was not sufficiently large for a comprehensive analysis despite the fact that no significant publication bias was detected in the meta-analysis. CD44 is not routinely studied in evaluating osteosarcoma. As such, those that are reporting upon its use are likely those that are interested specifically in CD44 specifically. Furthermore, it is difficult to perform a meta-analysis in subgroups on the basis of the current sample size. This might weaken the reliability of meta-analysis results. To get a more comprehensive evaluation of the prognostic role of CD44 expression in patients with osteosarcoma, more well-designed studies with larger sample sizes are needed.

Our current meta-analysis indicated that upregulation of CD44 is correlated with aggressive behaviors during osteosarcoma progression, including metastasis and unfavorable prognosis. However, the meta-analysis just showed a peripheral correlation between CD44 and the osteosarcoma, we still lack the causal relationship evidence to direct prove the function of CD44 in osteosarcoma. Therefore, we further design the in vitro experiment to knock out CD44 in highly metastatic human osteosarcoma cells by CRISPR-Cas9. Knocking out the CD44 gene by CRISPR-Cas9 in highly metastatic human osteosarcoma cells (MNNG/ 
HOS and 143B) further confirmed that CD44 plays crucial roles in human metastatic osteosarcoma cell migration and invasion. When CD44 was silenced, the proliferation and spheroid formation of osteosarcoma cells were inhibited, and the migratory and invasive functions were impaired in these cells. Therefore, the in vitro results could be a good explanation for the meta-analysis.

CD44 is a surface glycoprotein involved in cell-cell and cell-matrix interactions, cell motility, migration, matrix degradation, as well as the formation of tumor microenvironments [37]. CD44 expression is upregulated in advanced human breast tumors and is correlated with the level of the mesenchymal marker N-cadherin in these tumors [38]. CD44 is attributed to cells that have undergone an epithelial-mesenchymal transition, leading to tumor metastasis, and resulting in a worse prognosis for colon cancers [39]. In research on ovarian cancer, the level of CD44 is higher in the metastatic and recurrent cancer tissues than in the patient-matched primary tumor tissues [40, 41]. Moreover, a strong increase of CD44 expression was found in an ovarian cancer recurrence mouse model [41]. In vivo study showed mice with disruption of the $C D 44$ gene virtually ablated metastasis formation in osteosarcoma [42]. The CD44-HA interaction can also enhance lung metastasis and chemoresistance in osteosarcoma cells (SaoS-2 cell line) [30]. Additionally, in a mouse model that was established by inoculating osteosarcoma cells (POS-1 cell line) into the hind paws of mice, lung metastasis of osteosarcoma can be accelerated by upregulation of CD44 [43]. In line with these reports, we again confirmed that overexpression of CD44 plays a role in the metastatic behavior of osteosarcoma and may be a prognostic parameter of osteosarcoma. Moreover, we studied the function of CD44 using the human metastatic osteosarcoma cell lines and showed that CD44 expression contributes to the metastatic activity of MNNG/HOS and $143 \mathrm{~B}$ cells.

Previously, we observed that CD44 siRNA can efficiently suppress CD44 expression in osteosarcoma cell lines (KHOS and U2OS) [6]. However, RNAi based techniques can achieve only temporary and partial knockdown of transcribed mRNA, but not genomic DNA [6, 41]. In this study, we employed CRISPR-Cas9 to knockout CD44 in human metastatic osteosarcoma cell lines. The position of the CD44 sgRNA target is located on the first exon of the CD44 gene. CRISPR-Cas9 mediates cleavage of targets on DNA sites that are complementary to the $5^{\prime}-20$ nt region of the sgRNA that lies next to a PAM sequence. Compared with RNAi, the advantages of the CRISPR-Cas9 system include the fact that CRISPR-Cas9 is an exogenous system that does not compete with endogenous processes and that it functions at the DNA level to target transcripts, which results in knockdown or complete elimination of gene function [13, $14,44]$. Furthermore, the mechanism of CRISPR-Cas9 that directly blocks transcription is distinct from that of RNAi, for which knockdown of gene expression requires the destruction of already transcribed mRNAs prior to their translation $[41,45]$. In addition, CRISPR-Cas 9 could provide a larger targetable sequence space in which promoters of the gene may also be targeted [13, 14, 44]. Thus, CRISPR-Cas9 is a novel genome editing tool for switching gene expression at the DNA level.

CRISPR-Cas9 guided gene targeting is highly specific [46]. In our study, the results showed that CRISPR-Cas9 guided by CD44 sgRNA markedly decreased CD44 expression in osteosarcoma cell lines, whereas pEGFP-N3 had no effect on MNNG/HOS or 143B cell lines. Previous reports have found that suppressing CD44 by its specific siRNA dramatically decreases the migratory potentials and invasiveness of osteosarcoma cells [6]. We showed similar findings when knocking out CD44 by CRISPR-Cas9 in highly metastatic human osteosarcoma cells. More importantly, stable CD44 knockout osteosarcoma cell lines at the DNA level have been established, characterized, and intensively studied in the present work. These cell lines were cultured in a 3-D environment, which can mimic in vivo growth conditions. When CD44 was knocked out by CRISPR-Cas9, the formation of spheroids under 3 -D culture and the growth speed were significantly suppressed. At the same time, the spheroids' borders were smoother than those of the control. In a recent study, effective tumor shrinkage was observed in xenograft models by suppressing CD44 mRNA and protein [47]. 
It has been demonstrated that CD44 is a multi-structural cell surface receptor that binds HA with a particularly high affinity $[7,47,48]$. A study focused on ovarian cancer showed that peritoneal metastasis may be triggered when CD44 on the ovarian cancer cells surface binds to HA on mesothelial cells [49]. Another study has also demonstrated that CD44 expressed on the surface of colon cancer cells can facilitate binding endothelial P- or L-selectin and increase hematogenous spread of tumor [50]. Although the role CD44 expression plays in osteosarcoma metastasis is unknown, our results show that knockout of CD44 inhibits tumor cell migration, and invasion, which provides evidence that CD44 may directly participate in osteosarcoma progression.

In conclusion, we demonstrated that overexpression of CD44 could predict poor survival and metastasis in patients with osteosarcoma. CD44 plays crucial role in osteosarcoma migration, and invasion. These results suggest that developing new strategies to target CD44 in osteosarcoma may prevent metastasis and improved the clinical outcome of osteosarcoma patients.

\section{Acknowledgements}

This work was supported, in part, by the Gattegno and Wechsler funds, the Kenneth Stanton Fund, and the Jennifer Hunter Yates Foundation. Dr. Duan is supported, in part, through a grant from Sarcoma Foundation of America (SFA), a grant from National Cancer Institute (NCI)/National Institutes of Health (NIH), UO1, CA 151452-01, a pilot grant from Sarcoma SPORE/NIH, and a grant from an Academic Enrichment Fund of MGH Orthopaedics. Dr. Liu is supported by a scholarship from the Chinese Scholarship Council and the National Natural Science Foundation of China (81672176).

This study has been selected for presentation at the Orthopedic Research Society (ORS) 2018 Annual meeting (Mar. 10-Mar. 13, 2018), New Orleans.

\section{Disclosure Statement}

No conflict of interests exists.

\section{References}

1 Liu T, Zhang X, Zhang Q, Zhang X, Guo X: Total femoral reconstruction with custom prosthesis for osteosarcoma.World J Surg Oncol 2016;14:93.

2 Liu T, Guo X, Zhang X, Li Z, Zhang Q: Reconstruction with pasteurized autograft for primary malignant bone tumor of distal tibia. Bull Cancer 2012;99:87-91.

3 Li Z, Dou P, Liu T, He S: Application of Long Noncoding RNAs in Osteosarcoma: Biomarkers and Therapeutic Targets.Cell Physiol Biochem 2017;42:1407-1419.

-4 Hattinger CM, Pasello M, Ferrari S, Picci P, Serra M: Emerging drugs for high-grade osteosarcoma. Expert Opin Emerg Drugs 2010;15:615-34.

5 Meyers PA: Muramyl tripeptide (mifamurtide) for the treatment of osteosarcoma. Expert Rev Anticancer Ther 2009;9:1035-1049.

6 Gao Y, Feng Y, Shen JK, Lin M, Choy E, Cote GM, Harmon DC, Mankin HJ, Hornicek FJ, Duan Z: CD44 is a direct target of miR-199a-3p and contributes to aggressive progression in osteosarcoma. Sci Rep 2015;5:11365.

7 Chen L, Yao Y, Sun L, Zhou J, Miao M, Luo S, Deng G, Li J, Wang J, Tang J: Snail Driving Alternative Splicing of CD44 by ESRP1 Enhances Invasion and Migration in Epithelial Ovarian Cancer. Cell Physiol Biochem 2017;43:2489-2504.

-8 Fang M, Wu J, Lai X, Ai H, Tao Y, Zhu B, Huang L: CD44 and CD44v6 are Correlated with Gastric Cancer Progression and Poor Patient Prognosis: Evidence from 42 Studies. Cell Physiol Biochem 2016;40:567-578. 


\section{Cellular Physiology Cell Physiol Biochem 2018;46:1218-1230

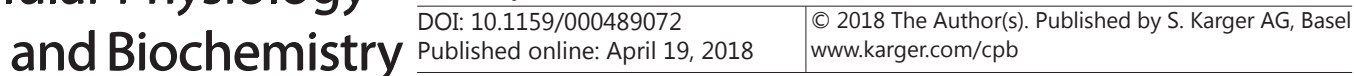

Liu et al.: Silence CD44 in Osteosarcoma Cells

9 Boldrini E, Peres SV, Morini S, de Camargo B: Immunoexpression of Ezrin and CD44 in patients with osteosarcoma. J Pediatr Hematol Oncol 2010;32:e213-217.

10 Liu Y, Wu Y, Gu S, Sun Z, Rui Y, Wang J, Lu Y, Li H, Xu K, Sheng P: Prognostic role of CD44 expression in osteosarcoma: evidence from six studies. Diagn Pathol 2014;9:140.

11 Kim HS, Park YB, Oh JH, Jeong J, Kim CJ, Lee SH: Expression of CD44 isoforms correlates with the metastatic potential of osteosarcoma. Clin Orthop Relat Res 2002;396:184-190.

12 Haidich A.B.: Meta-analysis in medical research. Hippokratia 2010; 14:S29-37.

13 Barrangou R: RNA events. Cas9 targeting and the CRISPR revolution. Science 2014;344:707-708.

14 Liu T, Li Z, Zhang Q De Amorim Bernstein K, Lozano-Calderon S, Choy E, Hornicek FJ, Duan Z: Targeting ABCB1 (MDR1) in multi-drug resistant osteosarcoma cells using the CRISPR-Cas9 system to reverse drug resistance. Oncotarget 2016;7:83502-83513.

15 Liu T, Shen JK, Li Z, Choy E, Hornicek FJ, Duan Z: Development and potential applications of CRISPR-Cas9 genome editing technology in sarcoma. Cancer Lett 2016;373:109-118.

-16 Stroup DF, Berlin JA, Morton SC, Olkin I, Williamson GD, Rennie D, Moher D, Becker BJ, Sipe TA, Thacker SB: Meta-analysis of observational studies in epidemiology: a proposal for reporting. Meta-analysis of Observational Studies in Epidemiology (MOOSE) group. JAMA 2000;283:2008-2112.

17 Tierney JF, Stewart LA, Ghersi D, Burdett S, Sydes MR: Practical methods for incorporating summary timeto-event data into meta-analysis. Trials 2007;8:16.

18 Parmar MK, Torri V, Stewart L: Extracting summary statistics to perform meta-analyses of the published literature for survival endpoints. Stat Med 1998;17:2815-2834.

19 Lau J, Ioannidis JP, Schmid CH: Quantitative synthesis in systematic reviews. Ann Intern Med 1997;127:820-826.

20 Higgins JP, Thompson SG: Quantifying heterogeneity in a meta-analysis. Stat Med 2002;21:1539-1558.

-21 Mantel N, Haenszel W: Statistical aspects of the analysis of data from retrospective studies of disease. J Natl Cancer Inst 1959;22:719-748.

-22 DerSimonian R, Laird N: Meta-analysis in clinical trials. Control Clin Trials 1986;7:177-188.

23 Begg CB, Mazumdar M: Operating characteristics of a rank correlation test for publication bias. Biometrics 1994;50:1088-1101.

24 Egger M, Davey Smith G, Schneider M, Minder C: Bias in meta-analysis detected by a simple, graphical test. BMJ 1997;315:629-634.

25 McAllister RM, Gardner MB, Greene AE, Bradt C, Nichols WW, Landing BH: Cultivation in vitro of cells derived from a human osteosarcoma. Cancer 1971;27:397-402.

26 Rhim JS, Putman DL, Arnstein P, Huebner RJ, McAllister RM: Characterization of human cells transformed in vitro by N-Methyl-N'-nitro-N-nitrosoguanidine. Int J Cancer 1977;19:505-510.

27. Hensler PJ, Annab LA, Barrett JC, Pereira-Smith OM: A gene involved in control of human cellular senescence on human chromosome 1q. Mol Cell Biol 1994;14:2291-2297.

-28 Luu HH, Kang Q Park JK, Si W, Luo Q, Jiang W, Yin H, Montag AG, Simon MA, Peabody TD, Haydon RC, Rinker-Schaeffer CW, He TC: An orthotopic model of human osteosarcoma growth and spontaneous pulmonary metastasis. Clin Exp Metastasis 2005;22:319-329.

29 Duan Z, Zhang J, Ye S, Shen J, Choy E, Cote G, Harmon D, Mankin H, Hua Y, Zhang Y, Gray NS, Hornicek FJ: A-770041 reverses paclitaxel and doxorubicin resistance in osteosarcoma cells. BMC Cancer 2014;14:681.

-30 Gvozdenovic A, Arlt MJ, Campanile C, Brennecke P, Husmann K, Li Y, Born W, Muff R, Fuchs B: CD44 enhances tumor formation and lung metastasis in experimental osteosarcoma and is an additional predictor for poor patient outcome. J Bone Miner Res 2013;28:838-847.

31 Deng Z, Niu G, Cai L, Wei R, Zhao X: The prognostic significance of CD44V6, CDH11, and beta-catenin expression in patients with osteosarcoma. Biomed Res Int 2013;2013:496193.

-32 Ma Q, Zhou Y, Ma B, Chen X, Wen Y, Liu Y, Fan Q, Qiu X: The clinical value of CXCR4, HER2 and CD44 in human osteosarcoma: A pilot study. Oncol Lett 2012;34:797-801.

33 Peng TS, Qiu JS, Wu HX, Liang HZ, Luo CQ: Expressions of CD44s, MMP-9, and Ki-67: possible association with invasion, metastasis, and recurrence of osteosarcoma. Ai Zheng 2002;21:745-750.

-34 Kuryu M, Ozaki T, Nishida K, Shibahara M, Kawai A, Inoue H: Expression of CD44 variants in osteosarcoma. J Cancer Res Clin Oncol 1999;125:646-652. 


\section{Cellular Physiology Cell Physiol Biochem 2018;46:1218-1230 \begin{tabular}{l|l} 
DOI: 10.1159/000489072 & $\begin{array}{l}\text { O 2018 The Author(s). Published by S. Karger AG, Basel } \\
\text { www.karger.com/cpb }\end{array}$ \\
\hline
\end{tabular}}

Liu et al.: Silence CD44 in Osteosarcoma Cells

-35 Hu WH, Chen AM, Guo FJ, Li F: The expression and clinical significance of KAI1 and CD44v6 protein in human osteosarcoma. The Chinese-German J Clin Oncol 2009;8:232-236.

-36 Zhang Y, Ding C, Wang J, Sun G, Cao Y, Xu L, Zhou L, Chen X: Prognostic significance of CD44V6 expression in osteosarcoma: a meta-analysis. J Orthop Surg Res 2015;10:187.

-37 Ponta H, Sherman L, Herrlich PA: CD44: from adhesion molecules to signalling regulators. Nat Rev Mol Cell Biol 2003;4:33-45.

-38 Brown RL, Reinke LM, Damerow MS, Perez D, Chodosh LA, Yang J, Cheng C: CD44 splice isoform switching in human and mouse epithelium is essential for epithelial-mesenchymal transition and breast cancer progression. J Clin Invest 2011;121:1064-1074.

39 Su Y.J, Lai HM, Chang YW, Chen GY, Lee JL: Direct reprogramming of stem cell properties in colon cancer cells by CD44. EMBO J 2011;30:3186-3199.

40 Miletti-González KE, Chen S, Muthukumaran N, Saglimbeni GN, Wu X, Yang J, Apolito K, Shih WJ, Hait WN, Rodríguez-Rodríguez L: The CD44 receptor interacts with P-glycoprotein to promote cell migration and invasion in cancer. Cancer Res 2005;65:6660-6667.

41 Gao Y, Foster R, Yang X, Feng Y, Shen JK, Mankin HJ, Hornicek FJ, Amiji MM, Duan Z: Up-regulation of CD44 in the development of metastasis, recurrence and drug resistance of ovarian cancer. Oncotarget 2015;11:9313-9326.

42 Weber GF, Bronson RT, Ilagan J, Cantor H, Schmits R, Mak TW: Absence of the CD44 gene prevents sarcoma metastasis. Cancer Res 2002;62:2281-2286.

43 Shiratori H, Koshino T, Uesugi M, Nitto H, Saito T: Acceleration of lung metastasis by up-regulation of CD44 expression in osteosarcoma-derived cell transplanted mice. Cancer Lett 2001;170:177-182.

-44 Ran FA, Cox D, Lin S, Barretto R, Habib N, Hsu PD, Wu X, Jiang W, Marraffini LA, Zhang F: Multiplex genome engineering using CRISPR/Cas systems. Science 2013;339:819-823.

-45 Qi LS, Larson MH, Gilbert LA, Doudna JA, Weissman JS, Arkin AP, Lim WA: Repurposing CRISPR as an RNAguided platform for sequence-specific control of gene expression. Cell 2013;152:1173-1183.

46 Zamore PD, Tuschl T, Sharp PA, Bartel DP: RNAi: double-stranded RNA directs the ATP-dependent cleavage of mRNA at 21 to 23 nucleotide intervals. Cell 2000;101:25-33.

47 Shah V, Taratula O, Garbuzenko OB, Taratula OR, Rodriguez-Rodriguez L, Minko T Targeted nanomedicine for suppression of CD44 and simultaneous cell death induction in ovarian cancer: an optimal delivery of siRNA and anticancer drug. Clin Cancer Res 2013;19:6193-6204.

48 Li CZ, Liu B, Wen ZQ, Li HY: Inhibition of CD44 expression by small interfering RNA to suppress the growth and metastasis of ovarian cancer cells in vitro and in vivo. Folia Biol (Praha). 2008;54:180-186.

49 Carpenter PM, Dao AV: The role of hyaluronan in mesothelium-induced motility of ovarian carcinoma cells. Anticancer Res 2003;23:3985-3990.

50 Napier SL, Healy ZR, Schnaar RL, Konstantopoulos K: Selectin ligand expression regulates the initial vascular interactions of colon carcinoma cells: the roles of CD44v and alternative sialofucosylated selectin ligands. J Biol Chem 2007;282:3433-3441. 